\title{
L'Amérique latine et les succès des politiques éducatives des tigres asiatiques
}

Latin America and the success of the education policies of the Asian tigers

América latina y las exitosas políticas docentes de los tigres asiaticos

Jose Weinstein et Macarena Hernández

Traducteur : Philippe Rabaté

\section{(2) OpenEdition}

Journals

Édition électronique

URL : https://journals.openedition.org/ries/4382

DOI : 10.4000/ries.4382

ISSN : 2261-4265

Éditeur

France Education international

Édition imprimée

Date de publication : 4 avril 2015

Pagination : 169-179

ISBN : 9782854206067

ISSN : $1254-4590$

Référence électronique

Jose Weinstein et Macarena Hernández, «L'Amérique latine et les succès des politiques éducatives des tigres asiatiques », Revue internationale d'éducation de Sèvres [En ligne], 68 | avril 2015, mis en ligne le 04 avril 2017, consulté le 02 juillet 2021. URL : http://journals.openedition.org/ries/4382 ; DOI : https://doi.org/10.4000/ries.4382 


\title{
L'Amérique latine et les succès des politiques éducatives des tigres asiatiques*
}

\author{
José Weinstein \\ Université Diego Portales, Chili \\ Macarena Hernández \\ Université Diego Portales, Chili
}

L'interprétation de notre propre réalité à l'aide de schémas étrangers ne fait que nous rendre chaque fois plus inconnus à nous-mêmes, chaque fois moins libres, chaque fois plus solitaires (Gabriel García Márquez) ${ }^{1}$

Au cours de ces dernières décennies, plusieurs pays asiatiques ont ébloui le monde par leur capacité de développement social et économique, qui s'est accompagnée en outre d'un investissement - dans le sens le plus vaste du terme puissant et tenace en éducation de la part de la société dans son ensemble (Birdsall et al., 1995). C'est ainsi que les bons indicateurs économiques sont allés de pair avec de remarquables résultats dans les enquêtes internationales. De fait, lors de la dernière campagne de la fameuse enquête PISA, des pays asiatiques en ont occupé les sept premières places, ce qui a conduit le journal El País à titrer l'un de ses articles de manière éloquente: «L'Asie gagne la course à l'éducation ».

La situation éducative des pays latino-américains se trouve aux antipodes de celle que nous venons d'esquisser. Si l'on observe les résultats de cette même enquête PISA, les huit pays latino-américains concernés se situent parmi les derniers rangs et le meilleur d'entre eux - le Chili - occupe la $51^{\mathrm{e}}$ place sur 65 pays participants. La distance sidérale qui sépare les uns des autres peut être illustrée à l'aide de la comparaison suivante : lorsqu'il a atteint l'âge de 15 ans, un élève chilien moyen a des résultats d'apprentissage équivalents à trois années d'études en moins par rapport à un élève moyen de Singapour.

Dans cet article, nous nous proposons d'examiner depuis l'Amérique latine ce qu'ont mené à bien certains pays asiatiques en pleine réussite sur le plan éducatif, pays que nous appellerons les tigres ${ }^{2}$, en nous focalisant sur une dimension particulière : leurs politiques éducatives. Ce choix n'est pas hasardeux mais

\footnotetext{
* Article traduit par Philippe Rabaté.

1. Citation extraite du discours prononcé par l'écrivain lors de la remise du prix Nobel de littérature (1982).

2. Pour être précis, la littérature spécialisée a donné à quatre économies le surnom de "tigres » ou " dragons " asiatiques : Hong-Kong, Singapour, Taïwan et la Corée du Sud. Dans cet article, nous ne considérons que Singapour et la Corée du Sud (en raison de leur participation à l'enquête Talis), et nous y ajoutons le Japon, dont le processus de développement économique a précédé celui des « tigres ».
} 
repose sur la conviction, de plus en plus répandue, que l'enseignement présentiel est le principal levier intra-scolaire de changement auquel les systèmes éducatifs peuvent - et doivent - avoir recours s'ils souhaitent entreprendre un processus de changement de la qualité de l'éducation (Unesco 2013 ; World Bank, 2012). Pour montrer la distance qui existe en matière d'enseignement entre les pays asiatiques et latino-américains, nous nous servirons des données récentes du rapport Talis 2013, spécialisé dans la thématique de l'enseignement. Finalement, nous achèverons notre réflexion en montrant les obstacles que les pays latino-américains doivent vaincre pour pouvoir progresser dans leurs politiques éducatives; les réalisations des tigres asiatiques peuvent constituer, à cet égard, un puissant point de référence, qui peut inspirer des changements qui devront toutefois tracer leurs propres parcours originaux.

\section{LES POLITIQUes ÉduCATIVES DES TIGRES ASIATIQUES}

L'importance des professeurs comme composante essentielle de la qualité de l'éducation est un thème sur lequel toutes les recherches en éducation s'accordent (OCDE, 2005 ; Barber et Mourshed, 2007 ; Daling Hammond et al., 2010). Les politiques éducatives des pays qui offrent les meilleurs résultats d'apprentissage des élèves sont caractérisées par une faisceau d'éléments communs parmi lesquels on peut dégager : 1) l'instauration d'une formation des enseignants de qualité qui intègre une diversité de contenus et de la mise en pratique ; 2) l'existence de programmes de formation et de tutorat pour les jeunes professeurs ; 3 ) l'offre d'opportunités variées de développement et d'apprentissage professionnel continu à l'intérieur et hors des écoles; et 4) une reconnaissance salariale compétitive et comparable à d'autres professions (Darling-Hammond et al., 2010).

Les réalités de pays comme Singapour, la Corée du Sud et le Japon s'alignent sur les principes qui viennent d'être exposés. Avec plus ou moins de force et d'intensité selon les cas, les politiques des tigres asiatiques se sont efforcé de promouvoir et d'assurer l'effectivité de ses professeurs, en agissant sur différentes dimensions comme la formation initiale, la formation continue et le développement professionnel, les conditions de travail et la carrière d'enseignant (Wang et al., 2003 ; Darling Hammond et al., 2010, Jensen et al., 2012 ; Ingersoll, 2007 ; Unesco, 2014). Derrière ces objectifs et ces buts affichés, ils ont déployé les stratégies suivantes :

- le maintien d'une offre de formation initiale des professeurs axée sur l'acquisition simultanée de connaissances, d'aptitudes pédagogiques et de formation pratique, et organisée au moyen d'une régulation centralisée et stricte de sa qualité - soit du fait de l'existence d'un opérateur unique qui agit sous couvert du ministère de l'éducation à Singapour, soit par le biais d'un système d'évaluation de la qualité des institutions de formations comme celui qui a été récemment créé en Corée du Sud, ou bien par l'introduction de différentes exigences que ces formations doivent satisfaire, au Japon; 
- l'existence de critères rigoureux d'entrée et/ou de sortie des programmes de formation qui mènent à une certification obligatoire pour enseigner ; ainsi, à Singapour par exemple, les candidats sont recrutés dans le premier tiers de chaque cohorte et doivent passer par un processus strict de sélection pour être admis, tandis qu'au Japon, ceux qui souhaitent devenir enseignants doivent passer un examen national pour être acceptés dans la formation et, qu'en Corée du Sud, c'est une épreuve finale qui mène à la certification ;

- l'instauration de programmes de formation continue obligatoires pour les nouveaux professeurs, fondés sur des méthodologies innovantes et essentiellement sur un tutorat dispensé par des professeurs expérimentés auprès des novices, comme on peut l'observer à Singapour avec le Structured Mentoring Programme, en Corée du Sud avec le programme provincial qui intègre des séminaires, des observations d'autres enseignants et l'appui des personnels de direction, ou au Japon avec l'initiative d'une année qui inclut l'appui de professeurs avancés à ceux qui s'initient à l'enseignement et d'autres activités comme des conférences et des visites d'autres écoles;

- l'offre d'une variété d'opportunités de développement professionnel, autant à l'intérieur comme à l'extérieur des écoles, qui atteignent 100 heures de formation en service à Singapour et 180 heures en Corée du Sud, à quoi s'ajoute la promotion d'autres pratiques d'apprentissage collaboratif entre collègues, comme les fameuses lesson studies au Japon;

- la mise en place de conditions de travail positives, avec un nombre moindre d'heures consacrées à l'enseignement que dans d'autres pays, des salaires compétitifs au sein du marché, et une stabilité dans le monde du travail due à l'obtention d'une titularisation automatique à Singapour et en Corée du Sud, ou bien au terme d'un année de stage dans le cas du Japon;

- l'établissement d'une carrière pour les enseignants, avec des opportunités de promotion horizontale et/ou verticale, dont les possibilités d'ascension sont déterminées par le résultat lors des évaluations du travail accompli et par l'expérience professionnelle, et qui impliquent dans les trois pays considérés des augmentations de salaire progressives.

\section{CARACTÉristiques ET PRATIQUES DES ENSEIGNANTS en Asie et en Amérique latine}

Les résultats récents de l'enquête Talis $2013^{3}$ permettent de comparer les caractéristiques et les pratiques des enseignants dans les systèmes scolaires des tigres asiatiques que nous examinons ${ }^{4}$ en les comparant aux trois pays latinoaméricains qui ont participé à l'enquête : le Brésil, le Chili et le Mexique.

3. Talis : Teaching and Learning International Survey. Cette enquête, réalisée par l'OCDE, renseigne sur les conditions d'enseignement et d'apprentissage. L'enquête 2013 portait sur les professeurs exerçant dans le secondaire inférieur et incluait 34 pays participants.

4. Dans notre comparaison, nous n'incluons pas un pays asiatique qui a également participé à Talis 2013 en raison de ses résultats éducatifs : la Malaisie. 
En ce qui concerne la formation initiale, la quasi-totalité des enseignants asiatiques ont achevé un programme de formation des enseignants (en moyenne, 94,5\% pour les trois pays), tandis que, parmi les enseignants latino-américains, il existe un groupe minoritaire, toutefois important, qui n'est pas allé au terme de ce programme et qui ne jouit pas d'une formation spécialisée (en moyenne, $25,6 \%$ pour les trois pays et, pour ce qui est du Mexique, près de $40 \%$ des enseignants se trouve dans ce cas de figure).

Les différences renvoient également à des éléments inclus dans ces programmes. Ainsi, dans les pays asiatiques, plus de $75 \%$ des professeurs déclarent que ces derniers comprennent une formation en contenus, en pédagogie et en mise en pratique dans toutes les matières enseignées. Dans les pays latinoaméricains, en revanche, $63 \%$ en moyenne des enseignants indiquent que ces contenus sont intégrés, et seulement $58 \%$ que l'on intègre de la pédagogie et de la mise en pratique pour toutes les matières - tous ces chiffres se trouvent en-dessous de la moyenne des pays de l'OCDE.

Ces différences dans la préparation s'approfondissent au moment de l'entrée dans le métier. L'information rapportée par Talis 2013 rend compte de réalités contrastées en matière de programmes formels de formation continue pour les professeurs novices - ce qui se traduit par le fait que, tandis qu'une ample majorité des enseignants asiatiques (presque $90 \%$ ) a participé à des activités de formation alors qu'ils occupaient leur premier poste, ceci constitue une expérience minoritaire pour les professeurs latino-américains.

Cette distance est également observable dans le développement professionnel que connaissent les deux groupes de professeurs dès qu'ils sont en service. Sur ce plan, les différences tendent à être davantage qualitatives (portant sur le type d'activité) que quantitatives (quantité de temps consacrée à chacune d'entre elle ou nombre d'activités réalisées). En effet, dans tous les pays considérés, les enseignants disent avoir participé très majoritairement aux activités de formation ou d'entraînement professionnel au cours des douze derniers mois. Cependant, parmi les pays asiatiques, les activités les plus innovantes, basées sur l'apprentissage entre pairs et de type plus expérimental - telles les rencontres de chercheurs, l'observation et les visites d'autres écoles, la participation à des réseaux ou l'appui de tuteurs - tendent à avoir une importance bien supérieure.

De même, la coopération entre les enseignants orientée vers le développement professionnel à l'intérieur des écoles varie fortement si l'on compare les tigres asiatiques et les pays latino-américains étudiés. Tandis qu'une écrasante majorité - une moyenne de 89,5 \% des enseignants asiatiques - déclare observer des classes et en rendre compte par la suite à d'autres professeurs de leur établissement scolaire au moins une fois par an, seule une minorité - 37,1\% - des enseignants latino-américains affirment le faire. 
Une autre ressource importante pour le développement professionnel des enseignants est le management pédagogique de leurs équipes de direction. Les cadres directifs des pays asiatiques étudiés montrent une meilleure formation préalable pour assumer leurs charges que leurs pairs latino-américains - tout particulièrement pour l'entraînement à la direction pédagogique (matière pour laquelle ils reçoivent une formation deux fois plus importante). En ce qui concerne leur développement professionnel dans le métier, on répète le modèle qui existe pour les enseignants : ils ont tous suivi des formations au cours des douze derniers mois, mais les asiatiques ont davantage tendu vers la réalisation d'activités innovantes, comme la participation à des réseaux d'apprentissage entre pairs, du tutorat ou des initiatives de recherche.

Graphique $n^{\circ} 1$.

Participation des cadres de direction à un réseau professionnel, un tutorat ou à des activités de recherche au cours des 12 derniers mois (en \%)

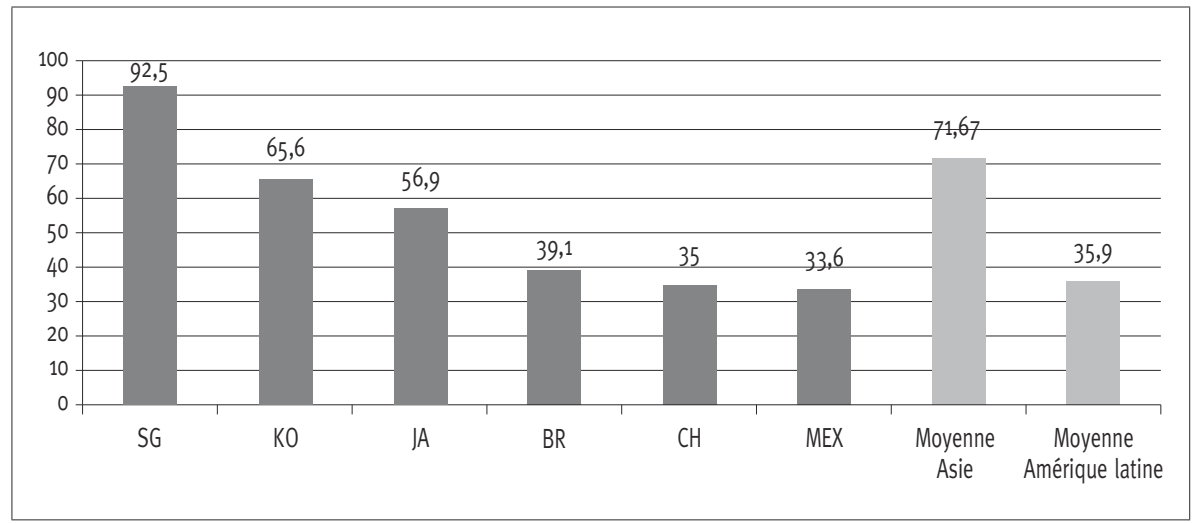

Source : élaboration personnelle à partir de Talis (2013)

$\mathrm{SG}=$ Singapour, $\mathrm{KO}=$ Corée du Sud, $\mathrm{JA}=$ Japon, $\mathrm{BR}=$ Brésil, $\mathrm{CH}=$ Chili, $\mathrm{MEX}=$ Mexique

Outre la formation initiale et la formation continue, une autre dimension révélatrice pour analyser la professionnalisation des enseignants dans un système scolaire déterminé repose sur leurs conditions de travail. En ce sens, les professeurs des tigres asiatiques ont une plus grande stabilité dans leur travail (avec un pourcentage très faible qui jouit d'un contrat à durée déterminée) et ils reçoivent de meilleurs salaires que leurs homologues latino-américains. Il convient d'ajouter qu'il y a non seulement une différence importante dans le salaire moyen, leurs rémunérations s'approchant beaucoup plus de celles d'autres professionnels dans leurs pays respectifs, mais également que ces revenus tendent à connaître une plus grande progression durant la carrière de l'enseignant et qu'ils peuvent atteindre des niveaux plus élevés, en termes absolus et relatifs, en haut de l'échelle (OCDE, 2014). 
L'extension horaire des contrats et l'usage assigné à ce temps au sein de la journée de travail des enseignants est un élément qui révèle une forte disparité. Tandis que les tigres asiatiques engagent les professeurs comme professionnels pour des journées complètes, les pays latino-américains ont l'habitude de le faire pour des journées d'emploi à temps partiel (33 heures par semaine en moyenne). Toutefois, la quantité de temps consacrée à faire effectivement cours pour les latino-américains... dépasse celle des asiatiques! Tandis qu'au Japon, en Corée du Sud et à Singapour, le plein exercice de la profession enseignante exige un temps important consacré à la préparation des cours, au dialogue avec les collègues, à l'accompagnement des élèves en-dehors des classes ou à l'investissement dans des activités extra-scolaires, au Brésil, au Chili et au Mexique ces activités sont très secondaires - le temps devant être consacré essentiellement à «faire cours $»^{5}$.

\section{Graphique $n^{\circ} 2$.}

Heures de travail totales déclarées par les professeurs et proportion moyenne destinée à l'enseignement présentiel.

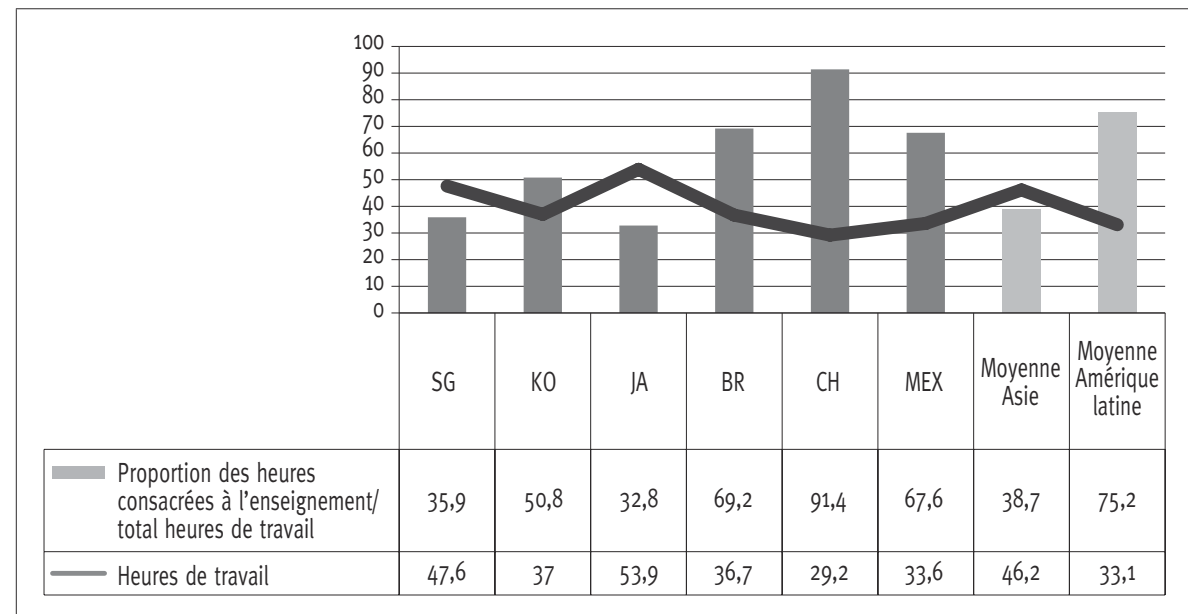

Source : élaboration personnelle à partir de TALIS (2013)

$\mathrm{SG}=$ Singapour, $\mathrm{KO}=$ Corée du Sud, JA = Japon, $\mathrm{BR}=$ Brésil, $\mathrm{CH}=$ Chili, $\mathrm{MEX}=$ Mexique

$\mathrm{Au}$ vu de ces antécédents, il n'est guère étonnant que les enseignants perçoivent de manière fort différente la valorisation sociale de leur profession. En ce qui concerne les tigres asiatiques, les professeurs se sentent majoritairement mis en valeur par leurs sociétés tandis que, dans les pays latino-américains analysés, une importante majorité pense le contraire et a le sentiment que leur labeur reçoit une reconnaissance sociale faible, voire nulle.

5. Étant donné que nombre de ces « autres activités » doivent obligatoirement avoir lieu, les enseignants latinoaméricains déclarent les effectuer hors de l'horaire pour lequel ils ont été engagés, ce qui donne lieu à une extension de leur journée qui ne fait l'objet d'aucune rémunération. 


\section{QUELS OBSTACLES L'AMÉRIQUE LATINE DOIT-ELLE SURMONTER POUR PROGRESSER?}

Affirmer que les pays d'Amérique latine devraient agir, pour leurs politiques éducatives, en suivant ce qu'ont pu mener à bien les tigres asiatiques voici plusieurs décennies, voilà qui semblerait une évidence. Il s'agit en effet d'augmenter drastiquement la qualité et la sélection de la formation initiale offerte par les universités, d'assurer un développement professionnel continu, pertinent, collaboratif et de qualité pour tous les enseignants, de construire des carrières professionnelles qui s'illustrent par l'attention portée à l'amélioration de l'éducation dans les salles de classe et qui offrent un salaire compétitif et des conditions de travail appropriées, et de mener à bien ces politiques de manière systématique, intégrale et avec une participation des professeurs eux-mêmes et de leurs organisations représentatives ${ }^{6}$.

Cela est facile à dire mais extraordinairement difficile à faire. L'enseignement et les enseignants de la région paraissent résister extraordinairement au changement, tant est faible la progression en termes d'effectivité professorale (Bruns et Luque, 2014). À quoi tient cette difficulté ? Pourquoi ne pas copier telles quelles ces politiques? Quels sont ces obstacles formidables que les politiques éducatives n'ont pas réussi à surmonter? Ils semblent relever au moins de quatre types différents : les coûts financiers, les capacités institutionnelles, les entraves culturelles et la conduite politique du changement (Weinstein, 2013). La considération de ces facteurs s'ajoute aux caractéristiques structurelles du contexte socio-économique latino-américain - comme les revenus inférieurs par habitant ou la plus grande inégalité perceptible face à d'autres régions du monde -, qui introduisent des restrictions supplémentaires alors même qu'il s'agit de focaliser les efforts et de progresser dans les politiques éducatives.

En matière de coûts économiques et financiers, il est évident que le nombre considérable d'enseignants en fonction dans le système scolaire des pays latino-américains fait que presque chaque mesure instaurée a un impact substantiel sur les finances nationales. Ce poids fiscal des augmentations salariales des enseignants a pour conséquence que les améliorations des rémunérations (lorsqu'elles existent) tendent à être modérées ou peu durables dans le temps - il n'est en effet pas possible de mener à bien des changements majeurs dans ce champ à cause de la responsabilité fiscale et de délais fort contraints ${ }^{7}$. Le nombre considérable d'enseignants fait que les autres mesures non-salariales en

\footnotetext{
6. Un récent travail entrepris par l'Unesco (2013) a permis de faire s'accorder les autorités gouvernementales, les experts et les représentants syndicaux d'Amérique latine, non seulement sur les dimensions que devaient apporter les politiques éducatives mais également sur les mesures spécifiques que celles-ci doivent privilégier.

7. Le fait que certains pays connaissent actuellement une transition démographique accentuée et aient par conséquent besoin d'un plus grande nombre d'enseignants dans les prochaines années, peut constituer une véritable opportunité pour améliorer leurs conditions de travail et professionnaliser leur activité (Bruns et Luque, 2014).
} 
leur faveur ont également un coût considérable, comme il arrive avec l'offre de formation professionnelle au service de bonne qualité, avec l'attribution d'un temps plus important de service qui ne relève pas de l'enseignement dans leur journée de travail normale, ou avec l'instauration de régimes spéciaux de retraites et de cessation anticipée d'activité. En outre, ces mesures sont communément des sources de dépense récurrente, de sorte que, une fois adoptées, elles font partie de l'exercice budgétaire et des droits acquis des enseignants. Financer de nouvelles politiques éducatives est alors une décision politique structurelle qui implique que, par delà l'ensemble des urgences et des besoins existants dans d'autres secteurs et champs d'activité, on réalise une " option-pays » à moyen et long terme, à la manière d'une politique industrielle.

Toutefois, même si l'on met à disposition les ressources financières du pays, elles ne constituent pas une condition suffisante en soi. Le développement réussi d'un certain type de politique exige l'existence de capacités institutionnelles qui ne se développent ni rapidement ni linéairement (Levin et al., 2008) et qui, en Amérique, sont très faibles (Scartascini et al., 2010). Le cas de la formation initiale des enseignants peut illustrer ce point. On peut faire preuve de la plus grande volonté gouvernementale pour transformer les facultés d'éducation et la qualité professionnelle de ceux qui les intègrent. Cependant, que ce changement ait effectivement lieu est un processus plus complexe, qui exige de changer la structure même des programmes, de rénover le corps des professeurs, d'intégrer de nouvelles pratiques de recherche, d'établir des relations avec les écoles comme des «domaines cliniques » et d'adopter des normes de sortie qui garantissent les connaissances et compétences des futurs enseignants (Darling-Hammond, 2012), tout ce processus se faisant dans des universités qui revendiquent leur autonomie institutionnelle. On peut retrouver cette complexité quand il s'agit de développer les capacités institutionnelles au niveau des cadres directifs des écoles ou des ministres de l'enseignement nationaux ou régionaux - et nombreuses sont les analyses qui, au-delà du secteur éducatif, identifient ce manque comme une faiblesse plus marquée et généralisée au sein de l'appareil public des pays latino-américains (Scartascini et al., 2010).

Un autre obstacle important relève des entraves culturelles des différents acteurs éducatifs, à commencer par les maîtres d'école eux-mêmes qui s'élèvent contre le développement de certaines des politiques éducatives. La question de la distribution équitable des capacités enseignantes au niveau national est un bon instrument pour examiner cette question. Pour qu'une politique éducative accomplisse pleinement ses objectifs d'équité, elle devrait permettre que les meilleurs enseignants travaillent dans les environnements d'enseignement les plus complexes sur le plan socio-éducatif. Mais la situation est habituellement contraire : les écoles les plus désavantagées d'Amérique latine sont prises en charge par des professeurs insuffisamment préparés ou inexpérimentés, avec des taux très élevés de démission et de changement de poste, et 
ces écoles ont bien des difficultés à compléter l'équipe enseignante dont elles ont besoin (Bruns et Luque, 2014). Les bons enseignants - à l'exception de quelques exceptions heureuses, communément motivées par des raisons idéologiques et/ou religieuses - se retrouvent habituellement dans les écoles de classes moyennes et élevées, avec un meilleur statut et de meilleures conditions de travail. L'objectif de la politique éducative se heurte alors à des valeurs dominantes dans la société latino-américaine actuelle, et à la définition réelle (non discursive) de ce qui constitue une carrière professionnelle réussie - qui n'est pas différente en ce qui concerne l'enseignement de celle qui s'impose à d'autres professionnels dans des sociétés où priment une économie de marché. Ces entraves culturelles sont également perceptibles dans d'autres dimensions des politiques éducatives et touchent les enseignants eux-mêmes, comme il arrive avec le développement professionnel continu qui exige communément que les professeurs changent leurs pratiques et ouvrent la boîte noire de la salle de classe (Elmore, 2010), mais également les autres acteurs éducatifs et sociaux - il suffit de penser par exemple à la relation problématique qu'entretiennent nombre de familles populaires d'Amérique latine avec l'école, qui n'incitent ni ne poussent leurs enfants - alors qu'ils sont adolescents - à participer à l'enseignement formel, ce qui complique considérablement la tâche des enseignants (Murillo et Roman, 2010).

Un dernier obstacle a trait à la conduite politique du changement et à sa capacité à faire progresser ces politiques éducatives au niveau de la prise de décisions. Comme nous l'avons vu, le défi est vaste : on doit posséder une solide capacité directive pour développer des politiques qui exigent à la fois un apport fiscal significatif et un temps long, pour demander des changements dans les capacités existantes dans les institutions et pour aller à contre-courant de certaines manières de voir et de faire les choses qui se sont enracinées - parfois chez les enseignants eux-mêmes. On retrouve la même tension politique lorsque l'on choisit d'investir dans la carrière des enseignants, au lieu de le faire dans des réalisations physiques (infrastructures scolaires, jardins d'enfants, etc.) ou bien dans des initiatives d'appui direct aux élèves (alimentation, fournitures scolaires, livres, bourses), les bénéfices de ces deux dernières options étant en effet directement perceptibles par la population et les électeurs. La relation avec les puissants syndicats enseignants, fortement marqués idéologiquement dans la plupart des cas, est particulièrement difficile à gérer tant y règne la confrontation et rares sont les exemples de régions où se sont développées des alliances entre gouvernement et représentants du personnel enseignant, afin d'appuyer des politiques éducatives précises (Palamidessi, 2003 ; Weinstein, 2006). Cette indispensable capacité de conduite politique du changement n'est alors pas facile à obtenir et encore moins à conserver dans le temps. L'existence d'un décalage entre le temps politique limité, dont disposent les différents gouvernements pour mettre en pratique leurs programmes, et le temps long qui est techniquement nécessaire 
pour obtenir des avancées réelles dans les matières éducatives et enseignantes complexes, en particulier dans le changement des pratiques présentielles, constitue un frein à l'obtention de résultats réels ${ }^{8}$.

Malgré tout, le capacité politique à mener à bien une réforme éducative est le plus décisif des quatre facteurs mentionnés puisque, même si c'est une ressource rare - et, en ce sens, en disposer de manière suffisante constitue en soi un obstacle - c'est également la clef qui permettra potentiellement de se défaire de l'inertie existante, en réduisant les résistances et en permettant que les politiques éducatives constituent une sorte de levier dans les systèmes scolaires latino-américains.

En somme, les tigres asiatiques ont réussi à suivre un chemin bénéfique pour leur éducation qui, entre autres choses, est parvenu à instaurer stratégiquement des politiques éducatives de grand impact et de forte effectivité. Aujourd'hui, leurs citoyens, mais également leurs économies, récoltent les fruits d'une action menée avec une ardente patience et dans laquelle ils ont réussi à aligner la volonté politique sur la capacité technique, le travail gouvernemental sur le compromis social. Les pays latino-américains devraient connaître ces expériences couronnées de succès, ainsi que d'autres qui se sont développées à un niveau international pour, depuis celles-ci, construire leurs propres propositions afin d'atteindre des cours de qualité pour tous ses garçons, filles et jeunes, passage indispensable pour améliorer l'éducation. Il ne sera guère aisé de vaincre les formidables obstacles économico-financiers, de capacités institutionnelles, de «culture éducative » et de conduite politique du changement qui existent dans les pays de la région pour construire et développer ces politiques éducatives efficaces. Cela exigera le déploiement, de la part de l'État et de la société, d'une volonté ferme et focalisée de changement sur plusieurs décennies, mais également d'une capacité de rencontrer effectivement les réponses techniques et politiques appropriées face à des dilemmes difficiles, ainsi que de libérer les potentialités existantes chez leurs éducateurs et leurs communautés scolaires. Cela implique, en paraphrasant Gabriel García Márquez, d’interpréter notre réalité éducative avec nos propres schémas, en utilisant les expériences courageuses des autres pour apprendre, s'inspirer et enrichir nos propres propositions dans un monde et une éducation sans recettes...

8. L'une des manifestations de cette difficulté à gérer politiquement le secteur éducatif sont les changements fréquents de ministres dans le secteur. L'on a estimé que le temps moyen de maintien des ministres de l'éducation dans leurs fonctions ne dépasse pas un an et demi, ce qui en fait l'un des portefeuilles gouvernementaux qui connaît le plus grand renouvellement (Unesco, 2013). 


\section{BIBLIOGRAPHIE}

BARBER M., \& MOURSHED M. (2007): How the world's best-performing school systems come out on top, London : McKinsey and Company.

BIRDSALL N., ROSS D., SABOT R. (1995): Inequality and growth reconsidered: lessons from East Asia, The World Bank Economic Review, World Bank, Washington.

BRUNS B., LUQUE J. (2014): Great teachers: how to raise student learning in Latin America and The Caribbean, Washington, DC : Banco Mundial.

DARLING-HAMMOND L., CHUNG WEI R., ANDREE A. (2010) : How high-achieving countries develop great teachers, Research Brief, Stanford Center for Opportunity Policy in Education.

DARLING-HAMMOND L. (2012): Educar con calidad y equidad. Los dilemas del siglo XXI, Santiago : Centro de Innovación en Educación Fundación Chile.

ELMORE R. (2010): Mejorando la escuela desde la sala de clases. Serie Liderazgo Educativo, Santiago : Fundación Chile-Fundación CAP.

INGERSOLL R. (2007) : A Comparative Study of Teacher Preparation and Qualifications in Six Nations, Consortium for Policy Research in Education.

JENSEN B., HUNTER A., SONNEMANN J., BURNS T. (2012) : Catching up: learning from the best school systems in East Asia, Full report, Grattam Institute.

LEVIN B., GLAZE A., FULLAN M. (2008) : "Results Without Rancor or Ranking: Ontario's Success Story », Phi Delta Kappan, 90(4), p. 273-280.

América Latina y el Caribe : el debate actual, Paris : UNESCO y OREAL-UNESCO Santiago. MURILLO F.J., ROMAN M. (2010) : «Retos de la evaluación de la calidad de la educación en América Latina ", Revista Iberoamericana de Educación, 53, p. 97-120.

OECD (2005) : Teachers Matter Education and Training Policy: Attracting, Developing and Training Effective Teachers, Paris : OECD Publishing.

OECD (2014) : Education at a Glance 2014: OECD Indicators, Paris : OECD Publishing. PALAMIDESSI M. (2003) : Sindicatos docentes y gobiernos. Conflictos y diálogos en torno a la Reforma Educativa en América Latina, Santiago : PREAL.

SCARTASCINI C., SPILLER P., STEIN T., TOMMASI M. (2010) : « ¿Cómo se juega en América Latina? Instituciones políticas, procesos de negociación y políticas públicas ", dans Scartascini C., Spiller P., Stein T., Tommasi M. (eds), El juego político en América Latina ¿cómo se deciden las políticas públicas?, BID.

UNESCO (2013) : Antecedentes y criterios para la elaboración de politicas docentes en América Latina y El Caribe, Estrategia Regional Para Docentes, Santiago : UNESCO/ OREALC.

UNESCO (2014) : Education systems in ASEAN+6 countries: A comparative analysis of selected educational issues, Paris : UNESCO.

WANG A., COLEMAN A., COLEY R., PHELPS R. (2003) : Preparing Teachers Aroung the World, Policy information report, Princeton : Educational Testing Service.

WEINSTEIN J. (2006) : « Chile 2000: la negociación MINEDUC-Colegio de Profesores. Una visión personal ", dans Espínola V., Amorin A. (éd.), Sindicalismo docente y reforma educativa, Washington: BID.

WEINSTEIN J. (2013) : La esquiva política entre las volátiles políticas docentes. En UNESCO Temas críticos para formular nuevas politicas docentes en América Latina y el Caribe: el debate actual, Paris : UNESCO y OREAL-UNESCO Santiago.

World Bank (2012) : What matters most in teacher policies? A framework for building a more effective teaching profession. SABER (Systems Approach for Better Education Results), Washington: The World Bank. 
